\title{
Superconducting Synchrotrons
}

Some topics from a Seminar, held on 26-29 April, organized by the Rutherford Laboratory.

This 'Seminar on Superconducting Synchrotrons' brought together about forty specialists working on the problems of developing pulsed superconducting magnets for use in synchrotrons. All the Laboratories with a major effort in the field (Berkeley, Brookhaven, Karlsruhe, Rutherford, Saclay) were represented with the exception of the Radiotechnical Institute Moscow, whose people were unfortunately unable to attend, and there were a few people also from CERN and Frascati.

With such a small knowledgeable gathering, it was possible to get deep into the subject from the word go. Each session concentrated on a few topics and was opened by one of the participants spending a few minutes listing the important problems. Each group could then present their approach to the solutions. The flow of information was fast and furious with some stimulating conflicts. This way of conducting the Seminar was very successful and was helped along by the able Chairmanship of D.A. Gray during all the sessions.

The Seminar was held in Cozener's House on the banks of the river Thames in Abingdon which serves as a hostel for the Rutherford Laboratory. This charming, characteristically English, setting was particularly appreciated by the visitors from the continent and the USA. Another characteristically English feature was the presence of a large dog which padded occasionally into the lecture room and barked at the more optimistic statements. It was particularly excited by speakers from Brookhaven. No-one could discern whether the barks denoted approval or rejection.

However, the human beings present were confident of the future of superconducting synchrotrons. As usual, this confidence is stated differently on either side of the Atlantic. The Americans tend first to state what can be done and then list the problems which remain to be swept out of the way in order to do it. The Europeans tend first to list the problems and then come to what could be done when they are solved. This subtle difference means a lot when it comes to the 'public relations' exercise of presenting this exciting work to others.

Nevertheless both sides, when pushed to it, would say that superconducting synchrotrons can be built. There remain many problems to come to grips with (quite apart from the detailed work to arrive at an optimum magnet design, there are major items such as the refrigeration and helium transport systems which have had little attention so far) but none of them look insoluble. However, because there is much work still to do, there is one parameter which no-one could yet confidently feed into the design of a superconducting synchrotron. To be realistic, it happens to be the most important one... the cost. To generalize a reaction to the Seminar, the question is no longer whether a superconducting synchrotron can be built but whether the studies of the next few years will refine all aspects of the design so that it can be built at an acceptable cost.

\section{Magnet design}

All Laboratories are concentrating on the use of niobium-titanium as the superconductor in the form of thin filaments in a twisted composite conductor where the filaments are embedded in copper and coppernickel matrices (see, for example, vol. 10, page 48). For example, Rutherford have built cables of 7 or 19 twisted strands, each strand being about $0.35 \mathrm{~mm}$ diameter and having up to $1045 \mathrm{NbTi}$ filaments of $8 \mu \mathrm{m}$ diameter embedded (thus consisting of around $50 \%$ superconductor). Other Laboratories, particularly Brook- haven, prefer the conductor formed into flat braid. The aim is to have a maximum current density - with the Rutherford conductor of over $20 \mathrm{kA} /$ $\mathrm{cm}^{2}$ for fields of over $4.5 \mathrm{~T}$. (Others have rather similar aims $-30 \mathrm{kA} / \mathrm{cm}^{2}$ for $5 \mathrm{~T}$ at Saclay, over $20 \mathrm{kA} / \mathrm{cm}^{2}$ for $5 \mathrm{~T}$ at Berkeley.)

With cable, 'self-field' effects have to be watched (Rutherford once found current running in the wrong direction down the centre strand of a seven strand cable) but generally the indications are that losses due to self-field effects will be negligeable compared with other losses. (A calculation from Karlsruhe in one particular conductor configuration gave $0.1 \mathrm{~W} / \mathrm{m}$ as the self-field loss compared with $4 \mathrm{~W} / \mathrm{m}$ from hysterisis and $3 \mathrm{~W} / \mathrm{m}$ from eddy currents.)

Before leaving the conductor, it is worth reporting some promising work at Brookhaven on another type of superconductor - vanadium-gallium, $\mathrm{V}_{3} \mathrm{Ga}$, which can also be produced in thin filamentary form. It can climb to considerably higher fields (over $20 \mathrm{~T}$ ) than $\mathrm{NbTi}$ before going normal (losing its superconducting property) and its critical temperature (the temperature at which it goes normal) is $14.5 \mathrm{~K}$. This could give great savings in refrigeration costs running $\mathrm{V}_{3} \mathrm{Ga}$ at, say, $8 \mathrm{~K}$ rather than $\mathrm{NbTi}$ at 4 to $5 \mathrm{~K}$. Brookhaven are having enough $\mathrm{V}_{3} \mathrm{Ga}$ made to build a small magnet and Karlsruhe are also planning to study it. However, despite its enticing properties, it is unlikely that $V_{3} G a$ superconductor will be available on a commercial scale, as is $\mathrm{NbTi}$, for many years.

There are $a$ variety of approaches to potting the conductor in coils. Berkeley are averse to potting, maintaining that it leads to poor coil performance since it inhibits good cooling which they maintain is the 
Photograph of a model superconducting dipoie magnet built at Brookhaven. It uses niobiumtitanium superconductor composite in braid form with the conductor around a circular apertur in a $\cos \Theta$ distribution. Laminated iron closely surrounds the superconductor. Such magnets, to give $4 T$ in the aperture, are now being built $50 \mathrm{~cm}$ long.

A rather more complex design is shown in the drawing. It involves two layers of superconducting windings so as to achieve a field of $6 T$. Note the coil spacing wedges which spread the conductor around the aperture and the 'clean' configuration of the conductor at the ends of the magnets.

more important factor. This is not borne out by others who are trying different materials. Rutherford, where the approach is typically broadlybased and thorough, have had many coils through many cool-downs both without impregnation and with impregnation (using unfilled epoxy resin, filled epoxy resin, wax, ice, oil or nitrogen). 'Training' (progressive approach to the performance achieved with short samples of the conductor) appeared to be correlated with internal mechanical energy stored in the coil during fabrication and cooling. As the coils settled down mechanically their performance improved and all the potted coils reached over $90 \%$ of the short sample performance.

Rutherford are going for filled epoxy and will probably look at some inorganic potting materials also. Brookhaven have recently had some good results from a thermosetting plastic insulation (known as Polybondex 180) which has excellent low temperature properties.

Coil shapes to give the required field configuration in the magnet aperture come in two styles - constant current density, requiring appropriate distribution of the current carrying conductor (arranged in ' $\mathrm{V}$ ' shape each side of a circular aperture in the Saclay design and like an elaborate ' $U$ ' each side of an elliptical aperture in the Karlsruhe design) and $\cos \Theta$ distribution, where the conductor is spaced cylindrically around the aperture with the conductor on the median plane carrying higher current than those above and below. This latter is the favoured alternative at Brookhaven illustrated in both the photograph and sketch. It has by no means been proved to be the optimum design, and needs more work before required precisions are assured, but it appears to have a simplicity of construction (a $35 \mathrm{~cm}$ long, $5 \mathrm{~cm}$ aperture model was completely wound
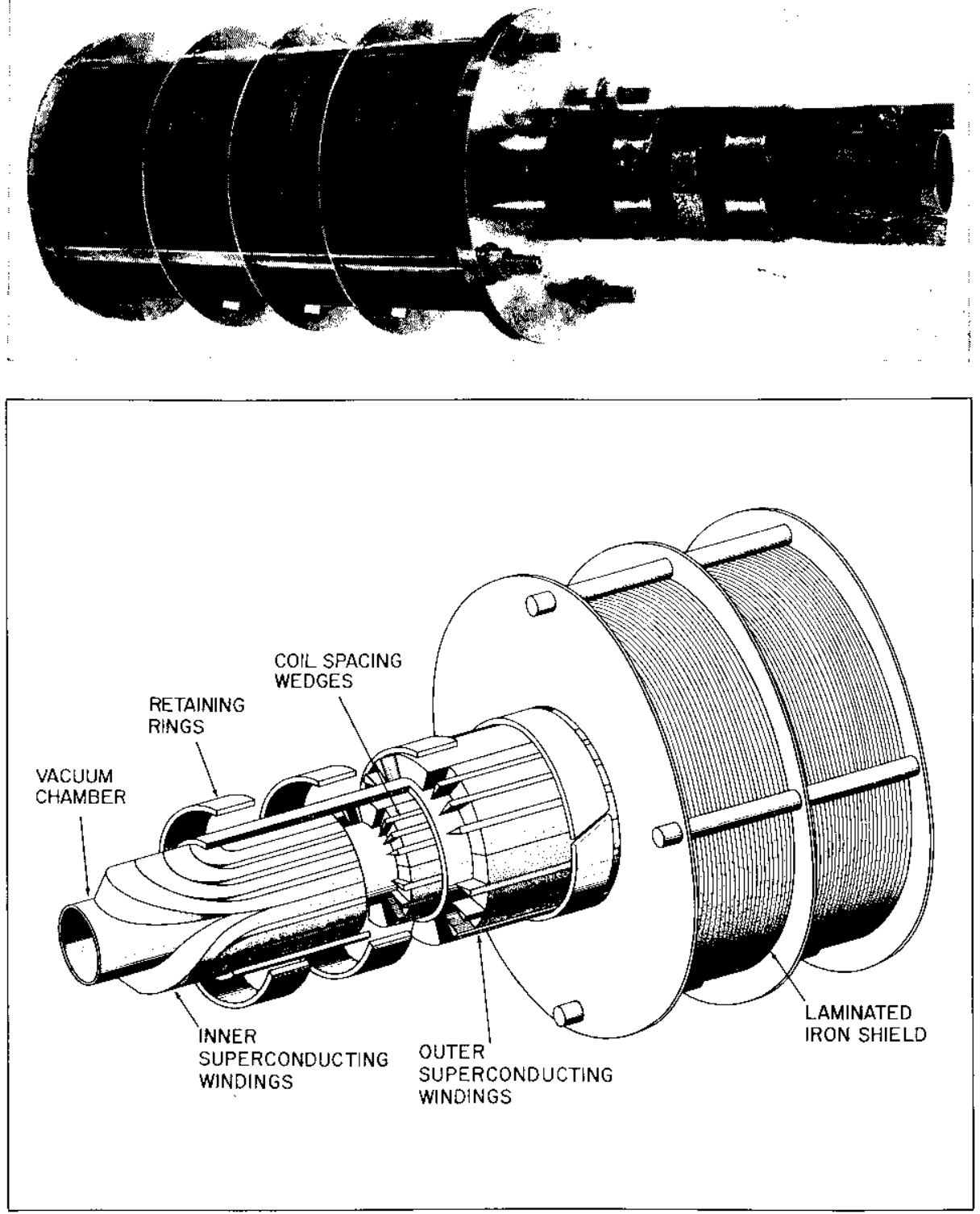

in less than four hours) which the design must ultimately have before magnets are produced commercially by the hundred for a high energy synchrotron.

The question of where to place the iron seems to be coming down in favour of iron as close as possible around the conductor. At Brookhaven, it is treated as a laminated iron core and an integrated part of the design, its thickness being selected to minimize the sextupole component of the field and its variations. It helps to keep stored energy down and can add over $1 \mathrm{~T}$ to the field in the aperture. It also serves as a mechanical clamp around the windings. The heat losses in the iron, which is within the cryostat, are not regarded as adding seriously to the refrigeration problems. Dipole magnets are however being built (e.g. at Saclay and Karlsruhe) with the iron used as a shield outside the cryostat to cut down external fields. Thus both options will be studied in the next few years.

Cryogenics, radiation problems, power supplies

Comparatively little attention has yet been given to the cryogenics problems on the large scale of a high energy synchrotron - there is little practical work to back up the calculations on the required refrigeration and on the costs likely to be involved. Estimates for the helium cooling system of a $1000 \mathrm{GeV}$ superconducting machine put the total losses (the static load from the dewars, etc. and from the helium distribution system plus the pulsed load from the heat produced in the magnets) at between 25 and $100 \mathrm{~kW}$ (cold watts) requiring up to $15 \mathrm{MW}$ (hot watts) of refrigeration, which is frightening but not prohibitive.

Associated problems - such as 
that of keeping down the total volume of helium (not the world's cheapest commodity), of transporting helium around kilometres of machine (perhaps inside a nitrogen cooled pipe, perhaps using the magnets themselves as the transfer line), of using one or several refrigeration stations, of magnet cryostat design (Karlsruhe are working on a plastic cryostat), of possibly enclosing superconducting power leads to the magnets inside the helium lines - all need more thought and some practical experience.

The effects of high doses of radiation on the materials likely to be used in the magnets also need attention. The worry about degradation of the superconductor itself which followed some measurements at Los Alamos (vol. 10, page 184) have been cleared but it would be comforting to have behind us some systematic high energy irradiations of proposed magnet materials. Some beam time at the present high energy machines could usefully be made available for this.

The required power supply to feed a high energy superconducting synchrotron is another frightening but not prohibitive feature. The amount of energy which will need to flow from the power supply to the magnet and back in a $1000 \mathrm{GeV}$ synchrotron is estimated at $150 \mathrm{MJ}$ on the basis of the Brookhaven magnet design and a factor of four higher on the basis of present European designs (Brookhaven plan for $20 \%$ lower field, iron close, lower operating temperature, more compact magnet - smaller aperture, less insulation). In either case, it is a lot of joules to pass around.

Several conventional motor-generator sets or static compensator systems (given a very healthy local electricity grid) could handle the problem. More appropriate, and potentially better if it can be proved in practice, is a novel superconducting power supply and energy store which is being studied at the Rutherford Laboratory. An analogue model of such a system is now in operation and it was one of the highlights of a tour of the superconducting work at the Laboratory to see the model in operation, with a pen recorder tracing out magnet cycles complete with injection platforms and flat-tops. An explanatory article on this power supply will appear in CERN COURIER in the next few months.

\section{Machine design studies}

A vital decision, when first sitting down to integrate individual components as discussed above into a machine, is to specify the required aperture. K. Green from Brookhaven puts it that you first work out your proton orbits and then put the hardware around them. Obviously, the smaller the good field aperture in the magnets the cheaper will be the magnets to build and to operate (probably reducing the refrigeration problems and certainly reducing the power supply problems since the stored energy goes up rapidly as the aperture is increased).

These considerations give added weight to the idea of building a superconducting synchrotron in association with an existing high energy machine which would serve as injector. Then the beam could be fed to the superconducting synchrotron already shrunk in size. (An interesting sideline debate on this topic concerned the shrinking in size of an accelerated beam above transition. Very recent results from the Brookhaven AGS indicate that it continues to shrink they finish with a virtually circular beam in configuration space of $1 \mathrm{~cm}$ diameter at $30 \mathrm{GeV}$ with $2 \times 10^{12}$ protons. At the CERN PS the effect is not clear and detection equipment is being refined to give more precise measurements.)

With high energy injection giving a smaller beam, the aperture in the superconducting ring can go down while still catering comfortably for injection and acceleration. However, this will be to no avail if more aperture is then needed for ejection. Work on slow ejection so far indicates that it might be this which dominates the aperture decision. In designing the machine it may be necessary to begin at the end and define the ejection requirements first. But if ejection does seem to dominate the aperture decision, maybe it is time to rethink ejection schemes.

The attitudes of the different Laboratories at present range from Brook haven maintaining that $5 \mathrm{~cm}$ diameter aperture is sufficient, to Saclay now building a magnet with $10 \mathrm{~cm}$ diameter aperture, with Karlsruhe now building a magnet with elliptical aperture $4 \times 7 \mathrm{~cm}^{2}$.

Another major decision is that of pulse repetition rate. On one side there is the philosophy promoted by R.L. Martin of Argonne to take the strain off the pulsing problems by running the machine as near d.c. as possible (10 s rise time, $100 \mathrm{~s}$ flat-top) keeping the physicists happy by accelerating $10^{14}$ or more protons per pulse. On the other side, most Laboratories are aiming for cycle times nearer to those to which we have become accustomed, i.e. in the $5 \mathrm{~s}$ range. This could need a superconducting power supply when the details of stored energy have been clarified.

Turning now to actual design studies : Berkeley are studying a $70 \mathrm{GeV}$ machine, $160 \mathrm{~m}$ in diameter with a peak field of $5 \mathrm{~T}$ pulsing at between once every 2.5 and once every $10 \mathrm{~s}$. The machine would be built in associ- 
ation with some existing facilities at LRL including initially $50 \mathrm{MeV}$ injection. A slow-cycling $2 \mathrm{GeV}$ superconducting booster could be added later. They are now designing and building superconducting magnets $30 \mathrm{~cm}$ to $1 \mathrm{~m}$ long.

Brookhaven have quite a detailed study of a 'Cold Magnet Synchrotron (CMS)', already in print (BNL 15430). They considered also the use of 'cryogenic' magnets (see CERN COURIER vol. 8 , page 186) but these are now taking second place to superconducting magnets. They have designed a superconducting conversion of the $30 \mathrm{GeV}$ AGS having another ring near the floor in the same tunnel. Parameters are - peak energy 112 $\mathrm{GeV}$, diameter $260 \mathrm{~m}$, magnetic field rising from 1 to $4 \mathrm{~T}$ injecting at $30 \mathrm{GeV}$ from the AGS. (If the $6 \mathrm{~T}$ magnets, mentioned above, are mastered the peak energy could be nearer $150 \mathrm{GeV}$.) The beam intensity is set at $10^{13}$ protons per pulse with a repetition rate of one pulse per $4 \mathrm{~s}$ (1.2 s acceleration, $1.6 \mathrm{~s}$ flat-top). They have also considered a $2000 \mathrm{GeV}$ superconducting synchrotron. The immediate effort is concentrated on building a set of magnets 30 to $50 \mathrm{~cm}$ long by the end of 1971 and, on the basis of experience with these magnets, to come to the detailed design and construction of magnets about $2 \mathrm{~m}$ long such as would be used in the machine.

Karlsruhe are thinking of a $1 \mathrm{GeV}$ 'table-top' model which could eventually serve as 'meson factory'). They are constructing $40 \mathrm{~cm}$ dipole magnets and hoping to have, sometime in 1972 , a $1 \mathrm{~m}$ magnet to give $5 \mathrm{~T}$ with a $2 \mathrm{~s}$ rise-time. They also have quite a broad programme of work on the associated problems - cryogenics, radiation, etc.

Rutherford have studied the possibility of a conversion, known as the SCS (superconducting synchrotron), of the $7 \mathrm{GeV}$ Nimrod. Using the exist- ing accelerator building, a machine with the following main parameters could be possible $-22 \mathrm{GeV}$ energy, $55 \mathrm{~m}$ diameter, peak field of $6 \mathrm{~T}$, injecting at $400 \mathrm{MeV}$ from a booster fed in turn by the existing $15 \mathrm{MeV}$ linac, accelerated intensity over $2 \times 10^{12}$ protons per pulse at a repetition rate of $6.75 \mathrm{~s}$. They have also considered a $50 \mathrm{GeV}$ machine removing the restriction of using existing buildings. Model magnets 50 to $100 \mathrm{~cm}$ long to give around $4.5 \mathrm{~T}$ are being designed and built.

Saclay have also proposed a superconducting conversion of their existing machine, the $3 \mathrm{GeV}$ Saturne. They maintain that before a very high energy machine could be undertaken, a 'pilot' machine of this kind should be built (other Laboratories might not insist on a full pilot machine but would certainly want to see a machine sector in operation). By about the end of 1971 Saclay hope to have a magnet $50 \mathrm{~cm}$ long, $10 \mathrm{~cm}$ aperture to give about $6 \mathrm{~T}$ with a $1.5 \mathrm{~s}$ rise-time.

A great deal of the effort at the three European Laboratories is going into the study of a superconducting synchrotron which might come about at the CERN Laboratory II. Options with regard to the use of pulsed superconducting magnets have been left open in the preliminary design of the CERN II machine (see, for example, vol. 10, page 110).

One option is that having started construction with conventional magnets (to give say $200 \mathrm{GeV}$ ) there could be sufficient space around the ring to slot in superconducting magnets to give at least double the energy. This idea of a single ring having both conventional and superconducting magnets looks rather less promising as the requirements of superconducting magnets become clearer. The need to go for the smallest possible aperture, for example, may prevent integration in a conventional ring. However, a ring in the same tunnel, possibly fed at high energy from a $200 \mathrm{GeV}$ conventional ring, is another alternative. A possible operating scheme which could take the pressure off the rise time of the superconducting magnets, if need be, would be to interleave the output pulses from the superconducting ring (fed by the conventional ring) and the conventional ring, thus feeding physics programmes at two energy ranges. But it is too early to pin things down.

The three European Laboratories have formed a collaboration known as GESSS (Group for European Superconducting Synchrotron Studies) which is effectively carrying out development work on behalf of CERN II and advising CERN II on the possibilities. GESSS meets at regular intervals to discuss problems and to pool information on progress. They have as their ultimate goal the design and construction of a $1000 \mathrm{GeV}$ superconducting machine at CERN II.

As the CERN II people, who will initially design a machine for several hundred $\mathrm{GeV}$ using conventional magnets, come together from the middle of this year there will no doubt also be close contact between them and GESSS. Several design decisions on the conventional machine could go a long way towards making life easier in any superconducting addition.

By the beginning of 1974, GESSS predicts that several superconducting pulsed magnets on a scale appropriate for a $1000 \mathrm{GeV}$ machine will have been built and put through their paces over several million cycles. By then the associated technologies will also be better understood and it should be possible to have a realistic stab at the cost. This information will then be fed into the balance pans at CERN II in discussing the future development of the Laboratory. 